\title{
Eficiência no uso dos nutrientes por espécies pioneiras crescidas em pastagens degradadas na Amazônia central
}

\author{
Carlos E. M. SILVA' ${ }^{1}$ José Francisco de C. GONÇALVES ${ }^{1}$, Ted R. FELDPAUSCH², Flávio J. LUIZÃO ${ }^{3}$, \\ Ronaldo R. MORAIS ${ }^{1}$, Glaudecy O. RIBEIRO ${ }^{1}$
}

\begin{abstract}
RESUMO
Nas últimas décadas, considerável parcela da floresta amazônica tem sido transformada em pastagens que, em função da baixa fertilidade natural dos solos e do manejo deficiente, decrescem rapidamente em produtividade e são abandonadas, dando lugar a uma sucessão secundária arbórea adaptada à baixa disponibilidade de nutrientes. O objetivo deste estudo foi obter informações sobre a eficiência no uso dos nutrientes por espécies arbóreas freqüentes nesses ambientes degradados, após calagem e aplicação de fosfato. O experimento realizado numa área de sucessão secundária com seis anos de idade, consistiu na aplicação de quatro tratamentos: controle; aplicação de fósforo $(\mathrm{P})$; aplicação de fósforo e calcário $(\mathrm{P}+\mathrm{Cal})$; e aplicação de fósforo, calcário e gesso $(\mathrm{P}+\mathrm{Cal}+\mathrm{G})$. As determinações das taxas de fotossíntese, bem como as coletas de amostras de solo e de folhas foram realizadas oito meses após a aplicação dos tratamentos. A aplicação do corretivo e do fosfato favoreceu o desempenho das espécies nos tratamentos $\mathrm{P}+\mathrm{Cal} \mathrm{e}$ $\mathrm{P}+\mathrm{Cal}+\mathrm{G}$. Bellucia grossularioides acumulou mais N, P e Zn, enquanto que Laetia procera acumulou mais Ca e Mn. Vismia japurensis foi a espécie mais eficiente no uso dos nutrientes, em função dos altos valores da taxa fotossintética; apresentou baixos teores foliares de P quando comparada com Bellucia grossularioides, sugerindo ser uma espécie bem adaptada a ambientes com baixa disponibilidade de nutrientes, como ocorre em áreas degradadas na Amazônia.
\end{abstract}

PALAVRAS-CHAVE

Áreas degradadas, sucessão secundária, trocas gasosas, calcário, fósforo, gesso.

\section{Nutrient use efficiency for pioneer species grown on abandoned pastures in central Amazonia}

\begin{abstract}
Large areas of Amazonian forest have been converted to pastures over the last two decades. Low soil fertility and mismanagement results in a rapid decline in net primary productivity leading the pastures to be abandoned, and woody vegetation adapted to low nutrient conditions colonize the areas. The objective of this study was to examine nutrient use efficiency, following liming (calcium) and phosphorus addition by three of the most frequent colonizing species. The experiment conducted on a six year-old secondary forest, consisted of four treatments: control; phosphorus addition (P); phosphorus and lime addition $(P+C a l)$; and phosphorus, lime and gypsum addition $(P+C a l+G)$. Leaf gas exchange, soil and leaf nutrient concentration were determined eight months after the treatment application. There was a significant response by species to the addition of phosphorus and lime $(P+C a l$ and $P+C a l+G)$. The species, Bellucia grossularioides accumulated more N, P and Zn in the leaves, while Laetia procera accumulated more Ca and Mn. The species Vismia japurensis had higher nutrient use efficiency, as a function of the higher photosynthetic rates. Vismia japurensis presented lower P concentrations than Bellucia grossularioides, suggesting that is well adapted to environments low in nutrients, as this species often occurs in degraded areas in Amazonia.
\end{abstract}

\section{KEY WORDS}

degraded area, secondary succession, gas exchange, gypsum, lime and phosphorus addition

\footnotetext{
${ }^{1}$ Laboratório de Fisiologia e Bioquímica Vegetal / Instituto Nacional de Pesquisas da Amazônia - INPA, Av. Efigênio Sales, nº 2239, Manaus, Amazonas, Brasil. CEP: 69066-970; Caixa postal: 476. Tel.: (092) 643-1880, cems@inpa.gov.br.

${ }^{2}$ Department of Crop and Soil Sciences / Cornell University (USA), trf2@cornell.edu.

${ }^{3}$ Coordenação de Pesquisas em Ecologia - Ciclagem de nutrientes / INPA, fluizao@inpa.gov.br.
} 


\section{INTRODUÇÃO}

Nas ultimas décadas, imensas áreas de florestas na Amazônia vêm sendo desmatadas e destinadas à formação de pastagens (Brandão et al., 2005). Sem manejo adequado e devido à baixa fertilidade natural dos solos amazônicos, a produtividade dessas áreas de pastagens declina rapidamente e, em conseqüência da sua baixa capacidade de suporte, são abandonadas, dando lugar a uma sucessão secundária emergente, composta principalmente por espécies pioneiras, como as dos gêneros Vismia, Bellucia e Laetia, adaptadas à baixa disponibilidade de nutrientes no solo (Monaco et al., 2003). Contudo, a regeneração natural da floresta nessas áreas é, muitas vezes, um processo lento, dificultado por uma combinação de alterações nos fatores primários, sejam eles de caráter biótico (competição entre plantas, ataque de patógenos, etc) ou abiótico (luz, temperatura, $\mathrm{CO}_{2}, \mathrm{H}_{2} \mathrm{O}$ e nutrientes) (Parrotta, 1992).

Dentre os fatores abióticos, a disponibilidade de nutrientes minerais destaca-se devido às funçôes específicas que os elementos minerais desempenham nas plantas, sendo essenciais para o seu crescimento e desenvolvimento (Drechsel \& Zech, 1993). Contudo, de maneira mais específica e sem tirar a importância dos demais macro e micronutrientes, o fósforo $(\mathrm{P})$ é um nutriente considerado determinante no crescimento das plantas, uma vez que tem considerável importância no metabolismo do carbono, bem como na formação de açúcares fosfatados (Grant et al., 2001). Esse elemento é absorvido pelas plantas principalmente na forma de $\mathrm{H}_{2} \mathrm{PO}_{4}$. Porém, no solo, sua abundância relativa é controlada pelo $\mathrm{pH}$ e sua concentração na solução do solo, geralmente é baixa, devido a sua rápida adsorção nas superfícies coloidais e a formação de precipitados como fosfatos de cálcio, magnésio, ferro ou de alumínio (van Raij et al., 2001). Dessa forma, em solos ácidos, como em geral ocorre na Amazônia, a concentração de P torna-se bastante limitante para o crescimento das plantas (Dias Filho, 1998). Nesse sentido, a correção da acidez do solo, com o uso do calcário, eleva o pH do solo, em função do decréscimo de íons de $\mathrm{H}^{+}$, promovendo o aumento da disponibilidade de P e também aumentando a concentração de $\mathrm{Ca}^{2+}$ e $\mathrm{Mg}^{2+}$ no solo (Albuquerque et al., 2003). Porém, esses efeitos se restringem à camada superficial do solo, onde o calcário foi incorporado, não trazendo grandes benefícios às camadas mais profundas. Por outro lado, a aplicação do gesso agrícola pode ser uma alternativa viável, pois, além de fornecer $\mathrm{Ca}^{2+} \mathrm{e}$ $\mathrm{SO}_{4}^{2-}$ ao solo, promove o movimento de cátions para regiões mais profundas do solo, aumentando os teores de bases e diminuindo a saturação por $\mathrm{Al}^{3+}$ (Marques et al., 1999).

Em áreas degradadas, as plantas são submetidas a situações extremas no que concerne à captação e utilização dos recursos primários (luz, $\mathrm{CO}_{2}, \mathrm{H}_{2} \mathrm{O}$ e nutrientes) e necessitam desenvolver estratégias adaptativas que favoreçam o estabelecimento e o crescimento nessas áreas durante o seu ciclo de vida (Puerta,
2002). Assim, vários aspectos ligados à eficiência fotossintética, como a utilização da energia luminosa, a assimilação de carbono, a regulação na absorção de água e de nutrientes, podem ser otimizados em função das diferentes demandas de cada espécie (Santos Jr., 2003). Para isto, o conhecimento dos mecanismos envolvidos na captação e na utilização dos recursos primários pelas diferentes espécies torna-se necessário para melhor compreender o quadro sucessional estabelecido em determinada área degradada. Desta forma, já está relativamente estabelecido na literatura que diferentes espécies pioneiras passam a dominar áreas de pastagens quando degradadas e abandonadas. No entanto, ainda não está devidamente elucidado como espécies de um mesmo grupo sucessional diferem funcionalmente para a assimilação e eficiência no uso dos nutrientes, quando submetidas a modificaçōes no ambiente edáfico. Portanto, o objetivo deste estudo foi investigar as respostas quanto à absorção e à eficiência no uso dos nutrientes por três das espécies pioneiras mais freqüentes (Vismia japurensis Reichardt, Bellucia grossularioides Triana e Laetia procera (Poepp.) Eichler) crescidas em áreas de pastagens abandonadas após diferentes tratamentos de correção da acidez do solo e adubação fosfatada na Amazônia central.

\section{MATERIAL E MÉTODOS}

\section{LOCAL DE ESTUDO}

O estudo foi realizado numa área de sucessão secundária sobre pastagens abandonadas, após seis anos, localizada no km 53 da rodovia BR-174, Estação Experimental da Embrapa Amazônia Ocidental, ao norte da cidade de Manaus ( ${ }^{\circ} 34^{\prime} \mathrm{S}$, $\left.60^{\circ} 02^{\prime} \mathrm{W}\right)$. O solo é classificado como Latossolo Amarelo com aproximadamente $80-85 \%$ de argila. O clima da região é tropical úmido do tipo $A m$, segundo a classificação de Köppen (1948), e a temperatura média é de $26,7^{\circ} \mathrm{C}$. A precipitação anual é de $2200 \mathrm{~mm}$, tendo março e abril como os meses mais chuvosos e os meses de agosto a outubro com menor precipitação (Lovejoy \& Bierregaard, 1990). A vegetação é classificada como floresta de terra firme sempre verde com dossel fechado, tendo as características estruturais da vegetação sucessional apresentadas por Feldpausch et al. (2004) e Feldpausch et al. (2005), como parte do projeto LBA - Experimento de Grande Escala da Biosfera Atmosfera na Amazônia.

O experimento foi implantado em janeiro de 2004, com a demarcação de quatro parcelas $(20 \times 40 \mathrm{~m})$ e seleção das espécies: V. japurensis, B. grossularioides e L. procera. Em seguida (fevereiro do mesmo ano), foram realizadas as aplicações de calcário dolomítico (2 ton ha ${ }^{-1}$ de $\mathrm{CaCO}_{3}$ ), gesso agrícola (1 ton ha ${ }^{-1} \mathrm{de}$ $\left.\mathrm{CaSO}_{4} 2 \mathrm{H}_{2} \mathrm{O}\right)$ e superfosfato triplo $\left(50 \mathrm{~kg} \mathrm{ha}^{-1} \mathrm{de}_{2} \mathrm{O}_{5}^{-}\right)$nos tratamentos. As determinaçôes das taxas fotossintéticas, bem como as coletas de amostras de solo e de folhas foram realizadas oito meses após a aplicação dos tratamentos. 


\section{FERTILIDADE DO SOLO}

Para a determinação da característica química e fertilidade do solo, sob cobertura das diferentes espécies pioneiras, foram coletadas três amostras de solo por tratamento, nas profundidades de $0-15,15-30 ; 30-45$ e $90-100 \mathrm{~cm}$. $\mathrm{O} \mathrm{pH}$ em água foi determinado conforme metodologia descrita por EMBRAPA (1999). O cálcio (Ca) e o magnésio $(\mathrm{Mg})$ foram determinados por espectrofotometria de absorção atômica (Perkin-Elmer $1100 \mathrm{~B}$ ), após extração por $\mathrm{KCl}_{1} \mathrm{~mol} \mathrm{~L}^{-1}$, sendo o alumínio (Al) determinado pelo método de titulação com $\mathrm{NaOH}$. O potássio $(\mathrm{K})$, ferro $(\mathrm{Fe})$, manganês $(\mathrm{Mn})$ e zinco $(\mathrm{Zn})$, também foram determinados por espectrofotometria de absorção atômica, após extração com solução de Mehlich 1. O fósforo (P) foi determinado por espectrofotometria do visível (Jenway 6105 UV/VIS), enquanto que o carbono $(\mathrm{C})$ e o nitrogênio $(\mathrm{N})$ foram determinados num analisador C/N (Fisions, NA 1500W).

\section{ESTADO NUTRICIONAL DAS PLANTAS}

Folhas das três espécies foram coletadas para a determinação dos teores de nutrientes foliares. As amostras foliares coletadas atendiam requisitos como: bom estado fitossanitário; completamente expandida; plena exposição ao sol; e situadas no terço médio da planta. $\mathrm{O}$ teor de $\mathrm{P}$ foi determinado por espectrofotometria do visível (Jenway 6105 UV/VIS), após digestão úmida $\left(\mathrm{HNO}_{3}+\mathrm{HClO}_{4}\right)$ do material vegetal. Os macronutrientes ( $\mathrm{K}, \mathrm{Ca}$ e $\mathrm{Mg}$ ) e os micronutrientes ( $\mathrm{Fe}, \mathrm{Mn}$ e $\mathrm{Zn}$ ) foram determinados por espectrofotometria de absorção atômica (Perkin-Elmer 1100B). O N total foi determinado pelo método de Kjeldahl, a partir do extrato obtido após digestão sulfúrica conforme metodologia descrita por EMBRAPA (1999).

\section{TROCAS GASOSAS}

As determinações das taxas de fotossíntese líquida $(A)$, da respiração $\left(R_{d}\right)$, da condutância estomática $(g)$ e de transpiração $(E)$, foram realizadas por meio de um analisador de gás infravermelho (IRGA) portátil, de sistema aberto, modelo LICOR 6400 (Santos Jr., 2003). As medições foram feitas em folhas maduras completamente expandidas, que apresentavam bom estado fitossanitário e estavam situadas no terço médio da copa. Para cada espécie, foi selecionada uma folha de uma planta para compor as repetiçóes, constituindo um total de três repetiçôes (indivíduos) por espécie e por tratamento (adubação). As respostas das variáveis fotossintéticas à intensidade luminosa foram determinadas para densidade de fluxo de fótons (PPFD) de $0 \mathrm{e}$ de $1500 \mu \mathrm{mol} . \mathrm{m}^{-2} \mathrm{~s}^{-1}$ com a câmara foliar ajustada para trabalhar com concentração de $\mathrm{CO}_{2}$, temperatura e vapor de $\mathrm{H}_{2} \mathrm{O}$ em torno de $380 \mu \mathrm{mol} \mathrm{mol}^{-1}, 31 \pm 1{ }^{\circ} \mathrm{C}$ e $21 \pm 1 \mathrm{mmol}^{\mathrm{mol}}{ }^{-1}$, respectivamente. A respiração no escuro $\left(R_{d}\right)$ foi determinada após uma adaptação de 15 minutos. Além disso, foram calculadas a fotorrespiração $(P$,$) de acordo com o método de Sharkey (1988).$

\section{DELINEAMENTO EXPERIMENTAL E ANÁLISE ESTATÍSTICA}

$O$ delineamento experimental utilizado foi o inteiramente casualizado, em esquema fatorial $4 \times 3$, sendo composto dos seguintes fatores: quatro tratamentos (controle; aplicação de fosfato - P; aplicação de fosfato + calagem - P+Cal; e aplicação de fosfato + calagem + gessagem $-\mathrm{P}+\mathrm{Cal}+\mathrm{G})$; e três espécies $(V$. japurensis, B. grossularioides e L. procera). Para a análise estatística dos dados obtidos foram feitas análises de variância, e as médias foram comparadas pelo teste de Tukey a $5 \%$ de probabilidade.

\section{RESULTADOS}

\section{FERTILIDADE DOS SOLOS}

Os valores de $\mathrm{pH}$ em $\mathrm{H}_{2} \mathrm{O}$ aumentaram nas camadas superficiais $(0-15$ e $15-30 \mathrm{~cm})$ dos tratamentos que sofreram correção da acidez do solo e aplicação de adubo fosfatado, quando comparados com o tratamento controle (Tabela 1). Esse aumento do valor do $\mathrm{pH}$ resultou numa diminuição significativa da concentração Al no solo somente na profundidade de $0-15 \mathrm{~cm}$ para o tratamento $\mathrm{P}+\mathrm{Cal}$.

As concentrações dos nutrientes, de modo geral, diminuíram com a profundidade (Tabela 1). Contudo, não se verificou um aumento na concentração de $\mathrm{C}$ e $\mathrm{N}$ nas camadas superficiais (zona de reação do calcário aplicado), quando comparado com o tratamento controle; as exceçôes foram a concentração de $\mathrm{C}$ na profundidade de $15-30 \mathrm{~cm}$ do tratamento $\mathrm{P}+\mathrm{Cal}+\mathrm{G}$ e a concentração de $\mathrm{C}$ e $\mathrm{N}$ na profundidade de $30-45 \mathrm{~cm}$ do tratamento P. Para o nutriente P, verificou-se um aumento nas concentraçōes na camada superficial $(0-15 \mathrm{~cm})$ dos tratamentos que receberam adubação fosfatada, em relação ao tratamento controle. Além disso, observaram-se, também, altos valores desse elemento na profundidade de $15-30 \mathrm{~cm}$, no tratamento $P$, e na profundidade de $30-45 \mathrm{~cm}$, no tratamento $\mathrm{P}+\mathrm{Cal}$. Nas camadas subsuperficiais $(15-30$ e $30-45 \mathrm{~cm}$ ) do tratamento $\mathrm{P}+\mathrm{Cal}+\mathrm{G}$, verificou-se uma elevada concentração de $\mathrm{K}$ em relação aos demais tratamentos, exceto quando comparado com o tratamento $\mathrm{P}$ na profundidade de $30-45 \mathrm{~cm}$, onde também foram encontrados altos valores de $\mathrm{K}$ em relação aos tratamentos $\mathrm{P}+\mathrm{Cal}$ e controle. As concentraçōes de Ca foram elevadas nas camadas de $0-15 \mathrm{e}$ $15-30 \mathrm{~cm}$ dos tratamentos $\mathrm{P}+\mathrm{Cal}$ e $\mathrm{P}+\mathrm{Cal}+\mathrm{G}$ e, também se observou maiores concentraçôes de Ca na camada de $30-45 \mathrm{~cm}$ do tratamento $\mathrm{P}+\mathrm{Cal}+\mathrm{G}$, quando comparado com os tratamentos $\mathrm{P}$ e controle. As concentraçōes de $\mathrm{Mg}$, foram mais altas na camada superficial do solo $(0-15 \mathrm{~cm})$ no tratamento $\mathrm{P}+\mathrm{Cal}$, quando comparadas com os demais tratamentos. Além disso, os valores $\mathrm{de} \mathrm{Mg}$ foram maiores nas camadas subsuperficiais do tratamento $\mathrm{P}+\mathrm{Cal}+\mathrm{G}$ quando comparados com os tratamentos $\mathrm{P}$ e controle. Para o micronutriente $\mathrm{Fe}$, as concentraçōes foram mais baixas na profundidade de $15-30 \mathrm{~cm}$ no tratamento controle do que nos demais tratamentos. Para o Mn, observou-se redução na 
concentração nas camadas subsuperficiais dos tratamentos que receberam aplicação de calcário, comparado com os tratamentos $P$ e controle. Em relação ao $Z n$, verificaram-se menores valores na camada de $0-15 \mathrm{~cm}$ dos tratamentos $\mathrm{P}, \mathrm{P}+\mathrm{Cal}$ e $\mathrm{P}+\mathrm{Cal}+\mathrm{G}$ comparado com o tratamento controle e, também, na camada de 90-100 cm do tratamento P+Cal.

\section{ESTADO NUTRICIONAL DAS PLANTAS}

Os teores foliares de $\mathrm{N}$ não diferiram entre os tratamentos. Entre as três espécies, $V$. japurensis apresentou menores concentrações de $\mathrm{N}$, em todos os tratamentos, do que as demais espécies estudadas (Figura 1).

Os maiores teores de P em B. grossularioides foram encontrados no tratamento $\mathrm{P}+\mathrm{Cal}$, significativamente maiores do que no controle. Além disso, verificaram-se maiores teores de $P$ para $B$. grossularioides no tratamento $\mathrm{P}+\mathrm{Cal}$, comparada com V.japurensis e $L$. procera. Os teores foliares de Ca da espécie $L$. procera foram maiores nos tratamentos que receberam aplicação de calcário $(\mathrm{P}+\mathrm{Cal}$ e $\mathrm{P}+\mathrm{Cal}+\mathrm{G})$ e no tratamento controle. $V$. japurensis apresentou maiores teores de Ca no tratamento $\mathrm{P}+\mathrm{Cal}+\mathrm{G}$ quando comparado com o tratamento controle. L. procera apresentou maiores teores foliares de $\mathrm{Mg}$ nos tratamentos com aplicação de calcário, seguida da B. grossularioides. Quando comparado com o tratamento controle, $L$. procera exibiu altos valores de Ca no tratamento $\mathrm{P}+\mathrm{Cal}$. L. procera apresentou elevados teores de $\mathrm{Fe}$ no tratamento controle. Os resultados dos teores foliares dos micronutrientes revelaram baixos teores de Mn para V.japurensis no tratamento P+Cal. L. procera foi a espécie que apresentou maiores teores de $\mathrm{Mn}$ em todos os tratamentos, quando comparada com as demais espécies estudadas. Para os teores de Zn, não se verificou diferença estatística entre os tratamentos, para nenhuma das espécies. Por outro lado, de modo geral, observaram-se os maiores e os menores teores de macro e micronutrientes para as espécies $B$. grossularioides e $L$. procera, respectivamente.

\section{TROCAS GASOSAS}

As três espécies estudadas apresentaram os maiores valores, para a maioria das medidas das trocas gasosas, sob os tratamentos $\mathrm{P}+\mathrm{Cal}$ e $\mathrm{P}+\mathrm{Cal}+\mathrm{G}$ (Tabela 2). No tratamento $\mathrm{P}+\mathrm{Cal}+\mathrm{G}$, por exemplo, $V$. japurensis exibiu maiores valores de $A, g_{s}, E, P$, enquanto que a variável $R_{d}$ não diferiu entre os tratamentos. Adicionalmente, no tratamento $\mathrm{P}+\mathrm{Cal}, B$. grossularioides e $L$. procera exibiram maiores valores para a maioria das variáveis analisadas, com exceção de $R_{d}$ para $L$. procera, onde os maiores valores foram observados no tratamento controle. Na comparação entre as espécies, $B$. grossularioides mostrou valores maiores para a maioria das variáveis, tanto no tratamento $\mathrm{P}+\mathrm{Cal}$ quanto no tratamento controle. Por outro lado, V.japurensis apresentou os maiores valores de $A, g_{s}, E, P_{r}$ nos tratamentos $\mathrm{Pe} \mathrm{P}+\mathrm{Cal}+\mathrm{G}$. A

Tabela 1 - Características químicas e de fertilidade dos solos nos diferentes tratamentos de adubação, em quatro profundidades do solo, numa floresta secundária na Estação Experimental da Embrapa, na Amazônia central.

\begin{tabular}{|c|c|c|c|c|c|c|c|c|c|c|c|}
\hline Tratamento & $\mathrm{pH}$ & Al & C & N & $\mathbf{P}$ & K & $\mathrm{Ca}$ & $\mathrm{Mg}$ & $\mathrm{Fe}$ & $\mathrm{Mn}$ & $\mathrm{Zn}$ \\
\hline & & meq $\%$ & _ $\mathrm{g} \mathrm{kg}-1$ & $\ldots$ & $\mathrm{mg} \mathrm{kg}-1$ & 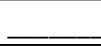 & cmolc kg- & & & $\mathrm{mg} \mathrm{kg}-1$ & \\
\hline \multicolumn{12}{|c|}{ Profundidade de $0-15 \mathrm{~cm}$} \\
\hline Controle & $4,3 \mathrm{a}$ & $1,4 \mathrm{a}$ & $26,4 \mathrm{a}$ & $1,7 \mathrm{a}$ & $1,7 \mathrm{a}$ & $0,12 a b$ & $0,10 \mathrm{a}$ & $0,12 \mathrm{a}$ & $353 a$ & $3,2 \mathrm{a}$ & $2,1 \mathrm{a}$ \\
\hline $\mathrm{P}$ & $4,5 \mathrm{~b}$ & $1,4 \mathrm{a}$ & $26,6 \mathrm{a}$ & $1,5 \mathrm{a}$ & $5,4 a b$ & $0,13 a b$ & $0,11 \mathrm{a}$ & $0,27 \mathrm{a}$ & 389 a & $4,7 \mathrm{~b}$ & $1,0 \mathrm{~b}$ \\
\hline $\mathrm{P}+\mathrm{Cal}$ & $4,9 \mathrm{c}$ & $0,9 \mathrm{~b}$ & $27,3 \mathrm{a}$ & $1,7 \mathrm{a}$ & $6,5 b$ & $0,11 \mathrm{a}$ & $1,16 \mathrm{c}$ & $0,71 \mathrm{~b}$ & $472 \mathrm{a}$ & $3,1 \mathrm{a}$ & $1,6 \mathrm{ab}$ \\
\hline $\mathrm{P}+\mathrm{Cal}+\mathrm{G}$ & $4,8 \mathrm{bc}$ & $1,4 \mathrm{a}$ & $28,4 \mathrm{a}$ & $1,6 \mathrm{a}$ & $6,6 \mathrm{~b}$ & $0,14 \mathrm{~b}$ & $0,61 \mathrm{~b}$ & $0,22 \mathrm{a}$ & $349 a$ & $4,5 b$ & $1,7 \mathrm{ab}$ \\
\hline \multicolumn{12}{|c|}{ Profundidade de $15-30 \mathrm{~cm}$} \\
\hline Controle & $4,3 \mathrm{a}$ & $1,0 \mathrm{a}$ & $14,0 \mathrm{a}$ & $0,9 \mathrm{a}$ & $2,7 \mathrm{a}$ & $0,06 \mathrm{a}$ & $0,05 \mathrm{a}$ & $0,07 \mathrm{a}$ & $284 \mathrm{a}$ & $2,5 \mathrm{a}$ & $0,7 \mathrm{a}$ \\
\hline$P$ & $4,3 \mathrm{a}$ & $1,1 \mathrm{a}$ & $15,6 a b$ & $1,0 \mathrm{~b}$ & $5,8 \mathrm{~b}$ & $0,08 \mathrm{a}$ & $0,07 \mathrm{a}$ & $0,10 \mathrm{c}$ & $445 \mathrm{~b}$ & $2,6 \mathrm{a}$ & $0,5 \mathrm{a}$ \\
\hline $\mathrm{P}+\mathrm{Cal}$ & $4,5 \mathrm{~b}$ & $1,0 \mathrm{a}$ & $14,9 a b$ & $0,9 \mathrm{a}$ & $1,4 \mathrm{a}$ & $0,07 \mathrm{a}$ & $0,25 \mathrm{~b}$ & $0,26 \mathrm{~d}$ & $472 \mathrm{~b}$ & $1,2 b$ & $1,0 \mathrm{a}$ \\
\hline $\mathrm{P}+\mathrm{Cal}+\mathrm{G}$ & $4,6 \mathrm{~b}$ & $0,8 \mathrm{a}$ & $18,0 \mathrm{~b}$ & $1,1 \mathrm{c}$ & $4,1 \mathrm{~b}$ & $0,11 \mathrm{~b}$ & $0,31 \mathrm{~b}$ & $0,21 \mathrm{~b}$ & $438 \mathrm{~b}$ & $1,5 b$ & $0,7 \mathrm{a}$ \\
\hline \multicolumn{12}{|c|}{ Profundidade de $30-45 \mathrm{~cm}$} \\
\hline Controle & $4,5 \mathrm{a}$ & $0,6 \mathrm{a}$ & $8,1 \mathrm{a}$ & $0,6 \mathrm{a}$ & $0,3 \mathrm{a}$ & $0,03 \mathrm{a}$ & $0,02 \mathrm{a}$ & $0,04 \mathrm{~b}$ & $244 a$ & $1,5 \mathrm{c}$ & $0,4 \mathrm{a}$ \\
\hline$P$ & $4,5 \mathrm{a}$ & $0,9 c$ & $13,2 b$ & $0,8 \mathrm{~b}$ & $1,0 \mathrm{a}$ & $0,06 \mathrm{c}$ & $0,03 \mathrm{a}$ & $0,04 \mathrm{~b}$ & $347 \mathrm{a}$ & $2,7 d$ & $0,2 \mathrm{a}$ \\
\hline $\mathrm{P}+\mathrm{Cal}$ & $4,5 \mathrm{a}$ & $0,9 \mathrm{c}$ & $10,5 a b$ & $0,7 \mathrm{a}$ & $2,7 \mathrm{~b}$ & $0,03 a b$ & $0,11 \mathrm{~b}$ & $0,08 \mathrm{a}$ & 267 a & $0,5 \mathrm{a}$ & $0,4 \mathrm{a}$ \\
\hline $\mathrm{P}+\mathrm{Cal}+\mathrm{G}$ & $4,7 \mathrm{a}$ & $0,8 \mathrm{~b}$ & $9,6 a b$ & $0,6 \mathrm{a}$ & $0,3 \mathrm{a}$ & $0,05 b c$ & $0,16 \mathrm{~b}$ & $0,06 \mathrm{a}$ & $190 \mathrm{a}$ & $1,0 \mathrm{~b}$ & $0,4 \mathrm{a}$ \\
\hline \multicolumn{12}{|c|}{ Profundidade de $90-100 \mathrm{~cm}$} \\
\hline Controle & $4,8 \mathrm{a}$ & $0,4 \mathrm{a}$ & $6,9 \mathrm{a}$ & $0,5 \mathrm{a}$ & $0,1 \mathrm{a}$ & $0,03 \mathrm{a}$ & $0,02 \mathrm{a}$ & $0,04 \mathrm{a}$ & $53 \mathrm{a}$ & $1,0 \mathrm{~b}$ & $0,5 \mathrm{ab}$ \\
\hline$P$ & 4,6 a & $0,7 \mathrm{c}$ & $8,1 \mathrm{a}$ & $0,6 \mathrm{~b}$ & $0,6 \mathrm{~b}$ & $0,03 \mathrm{a}$ & $0,04 \mathrm{a}$ & $0,02 \mathrm{a}$ & $51 \mathrm{a}$ & $2,1 \mathrm{c}$ & $0,8 \mathrm{bc}$ \\
\hline $\mathrm{P}+\mathrm{Cal}$ & $4,6 \mathrm{a}$ & $0,7 \mathrm{c}$ & $7,1 \mathrm{a}$ & $0,5 \mathrm{a}$ & $0,3 \mathrm{a}$ & $0,03 \mathrm{a}$ & $0,11 \mathrm{a}$ & $0,08 \mathrm{~b}$ & $22 b$ & $0,1 \mathrm{a}$ & $0,2 \mathrm{a}$ \\
\hline $\mathrm{P}+\mathrm{Cal}+\mathrm{G}$ & 4,8 a & $0,6 \mathrm{~b}$ & $6,6 \mathrm{a}$ & $0,5 \mathrm{a}$ & $0,1 \mathrm{a}$ & $0,02 \mathrm{a}$ & $0,09 \mathrm{a}$ & $0,06 \mathrm{~b}$ & $23 b$ & $0,5 \mathrm{ab}$ & $1,0 \mathrm{C}$ \\
\hline
\end{tabular}


espécie $V$. japurensis exibiu os menores valores de $R_{d}$ em quase todos os tratamentos, quando comparada com as demais espécies, enquanto que $L$. procera apresentou os menores valores de $A$ em todos os tratamentos.

\section{EFICIÊNCIA NO USO DOS NUTRIENTES}

De modo geral, sob os tratamentos com correção da acidez e aplicação de fosfato, as plantas apresentaram maiores valores da eficiência no uso dos nutrientes (EU[Nutriente]), quando comparadas com as plantas situadas no tratamento controle (Figura 2). A espécie V.japurensis, sob os tratamentos $\mathrm{P}+\mathrm{Cal}$ e $\mathrm{P}+\mathrm{Cal}+\mathrm{G}$, utilizou de modo mais eficiente a maioria dos nutrientes analisados, com destaque para $\mathrm{N}, \mathrm{P}$ e Mg, diferindo das demais espécies estudadas. B. grossularioides mostrou-se mais eficiente na
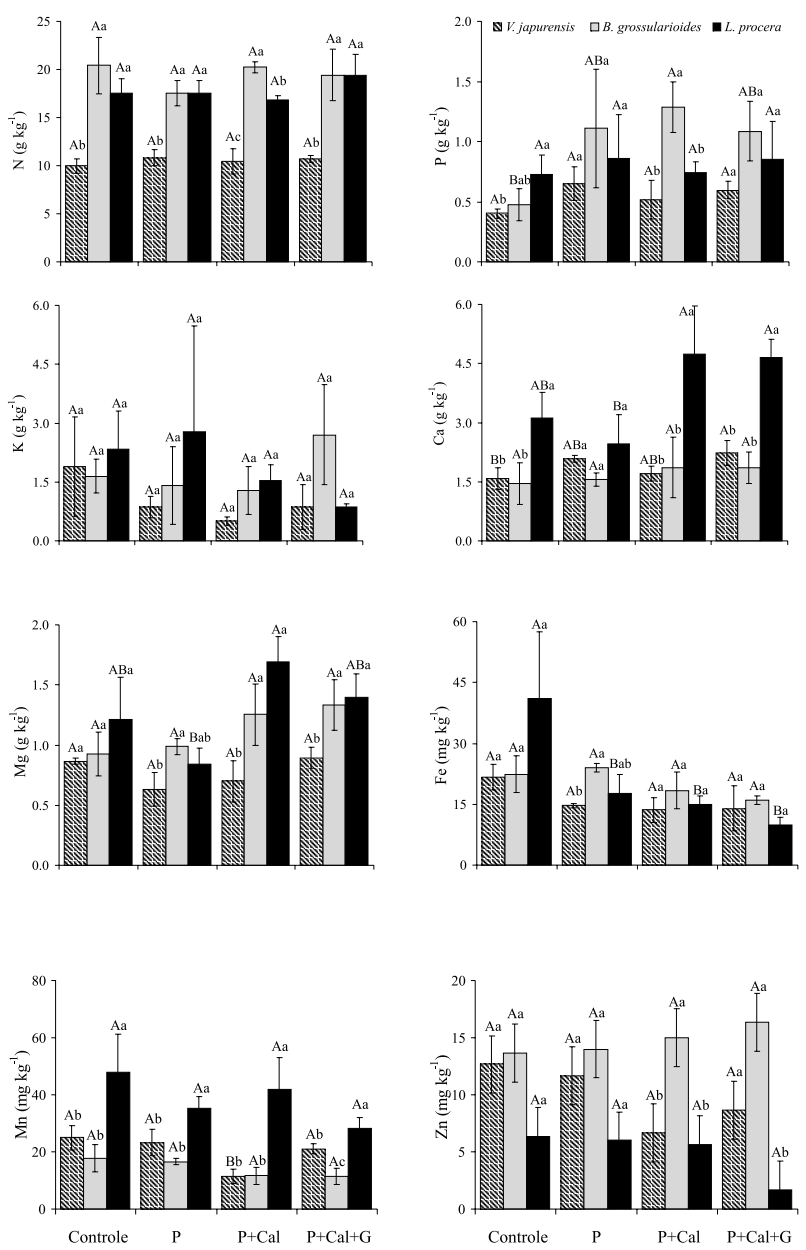

Figura 1 - Teores dos nutrientes foliares de três espécies pioneiras, submetidas a diferentes tratamentos de correção da acidez e adubação fosfatada, numa floresta secundária, na Estação Experimental da Embrapa na Amazônia central.

Os valores são médias $(n=3)$ e as linhas verticais indicam os desvios-padrão. Médias seguidas da mesma letra maiúscula, para os tratamentos, e da mesma letra minúscula, para espécies, não diferem pelo teste de Tukey a $5 \% . P=$ tratamento com aplicação de para espécies, não diferem pelo teste de Tukey a $5 \%$. $P$ = tratamento com aplicação de
fósforo; $P+C a l=$ tratamento com aplicação de fósforo e calcário; $P+C a l+G=$ tratamento com aplicação de fósforo, calcário e gesso agrícola. utilização de Ca e Mn. L. procera, apesar de não diferir para muitos nutrientes em relação a $B$. grossularioides, destacou-se na utilização eficiente do micronutriente $\mathrm{Zn}$.

\section{DISCUSSÃO}

\section{FERTILIDADE DOS SOLOS}

$\mathrm{O}$ aumento no valor do $\mathrm{pH}$ e a redução na concentração de $\mathrm{Al}$ nas camadas superficiais do solo $(0-15$ e $15-30 \mathrm{~cm})$ dos tratamentos $\mathrm{P}+\mathrm{Cal}$ e $\mathrm{P}+\mathrm{Cal}+\mathrm{G}$ podem ser atribuídos à aplicação de corretivos da acidez. Porém, a correção da acidez do solo e, conseqüentemente, a redução na concentração de $\mathrm{Al}$, não se evidenciou nas camadas mais profundas (abaixo de $30 \mathrm{~cm}$ ) do tratamento com aplicação de gesso, conforme era esperado. Isto talvez ocorreu porque a neutralização da acidez do subsolo normalmente é dificultada pelo aumento da retenção de cátions nas camadas superficiais, uma vez que os ânions resultantes da dissolução do calcário são, também, consumidos nas reações com outros cátions ácidos na camada de deposição do calcário (Albuquerque et al., 2003). Apesar da prática da calagem aumentar a eficiência na disponibilização do $\mathrm{N}$ do solo quando o

Tabela 2 - Fotossíntese líquida $(A)$, taxa de respiração $\left(R_{d}\right)$, fotorrespiração $\left(P_{r}\right)$, condutância estomática $\left(g_{s}\right)$, taxa de transpiração $(E)$ de espécies pioneiras, submetidas a diferentes tratamentos de correção da acidez do solo e adubação fosfatada, numa floresta secundária, na Estação Experimental da Embrapa, na Amazônia central.

\begin{tabular}{|c|c|c|c|c|}
\hline Variável & Tratamento & V. japurensis & B. grossularioides & L. procera \\
\hline \multirow{4}{*}{$\begin{array}{c}A \\
\left(\mu \mathrm{mol}\left(\mathrm{CO}_{2}\right)\right. \\
\left.\mathrm{m}^{-2} \mathrm{~s}^{-1}\right)\end{array}$} & Controle & $9,4 \pm 1,1 \mathrm{Ab}$ & $11,3 \pm 1,4 \mathrm{Ac}$ & $8,6 \pm 1,0 \mathrm{Aa}$ \\
\hline & $P$ & $13,4 \pm 1,0 \mathrm{Ba}$ & $12,5 \pm 0,5 \mathrm{Bb}$ & $8,9 \pm 0,9 \mathrm{Ac}$ \\
\hline & $\mathrm{P}+\mathrm{Cal}$ & $14,3 \pm 0,5 \mathrm{Ca}$ & $20,4 \pm 0,8 \mathrm{Db}$ & $14,1 \pm 0,9 \mathrm{Ca}$ \\
\hline & $\mathrm{P}+\mathrm{Cal}+\mathrm{G}$ & $18,7 \pm 0,4 \mathrm{Da}$ & $15,6 \pm 0,9 \mathrm{Cb}$ & $12,5 \pm 0,5 \mathrm{BC}$ \\
\hline \multirow{4}{*}{$\begin{array}{c}R d \\
\left(\mu \mathrm{mol}\left(\mathrm{CO}_{2}\right)\right. \\
\left.\mathrm{m}^{-2} \mathrm{~s}^{-1}\right)\end{array}$} & Controle & $1,7 \pm 0,5 \mathrm{Aa}$ & $2,0 \pm 0,7 \mathrm{Ab}$ & $2,3 \pm 0,4 \mathrm{Ab}$ \\
\hline & $P$ & $1,5 \pm 0,1 \mathrm{Aa}$ & $2,2 \pm 0,6 \mathrm{ABb}$ & $2,1 \pm 1,0 \mathrm{ABb}$ \\
\hline & $\mathrm{P}+\mathrm{Cal}$ & $1,7 \pm 0,3 \mathrm{Aa}$ & $2,6 \pm 1,1 \mathrm{Bb}$ & $1,8 \pm 0,6 \mathrm{BCa}$ \\
\hline & $\mathrm{P}+\mathrm{Cal}+\mathrm{G}$ & $1,7 \pm 0,7 \mathrm{Aa}$ & $1,9 \pm 0,6 \mathrm{Aa}$ & $1,5 \pm 0,6 \mathrm{Ca}$ \\
\hline \multirow{4}{*}{$\begin{array}{c}P r \\
\left(\mu \mathrm{mol}\left(\mathrm{CO}_{2}\right)\right. \\
\left.\mathrm{m}^{-2} \mathrm{~s}^{-1}\right)\end{array}$} & Controle & $1,7 \pm 0,1 \mathrm{Aa}$ & $2,0 \pm 0,4 \mathrm{Aab}$ & $2,3 \pm 0,9 \mathrm{ABb}$ \\
\hline & $P$ & $2,4 \pm 0,2 \mathrm{Ba}$ & $2,1 \pm 0,5 \mathrm{Ab}$ & $1,8 \pm 0,2 \mathrm{Cc}$ \\
\hline & $\mathrm{P}+\mathrm{Cal}$ & $2,4 \pm 0,1 \mathrm{Ba}$ & $3,5 \pm 0,1 \mathrm{Cc}$ & $2,5 \pm 0,1 \mathrm{Ab}$ \\
\hline & $\mathrm{P}+\mathrm{Cal}+\mathrm{G}$ & $3,1 \pm 0,1 \mathrm{Ca}$ & $2,7 \pm 0,1 \mathrm{Bb}$ & $2,0 \pm 0,1 \mathrm{BCC}$ \\
\hline \multirow{4}{*}{$\begin{array}{c}g s \\
\left(\mathrm{mmol}^{-}\left(\mathrm{H}_{2} \mathrm{O}\right)\right. \\
\left.\mathrm{m}^{-2} \mathrm{~s}^{-1}\right)\end{array}$} & Controle & $38,2 \pm 4,6 \mathrm{Ab}$ & $48,2 \pm 14 \mathrm{Aa}$ & $11,8 \pm 2,1 \mathrm{Ac}$ \\
\hline & $P$ & $53,9 \pm 11 \mathrm{Ba}$ & $41,6 \pm 12 \mathrm{Ab}$ & $29,6 \pm 7,2 \mathrm{BC}$ \\
\hline & $\mathrm{P}+\mathrm{Cal}$ & $50,8 \pm 4,9 \mathrm{Ba}$ & $157,4 \pm 28 \mathrm{Bb}$ & $59,1 \pm 17 \mathrm{Da}$ \\
\hline & $\mathrm{P}+\mathrm{Cal}+\mathrm{G}$ & $106 \pm 6,6 \mathrm{Ca}$ & $44,5 \pm 6,5 \mathrm{Ab}$ & $46,8 \pm 3,8 \mathrm{Cb}$ \\
\hline \multirow{4}{*}{$\begin{array}{c}E \\
\left(\mathrm{mmol}^{\left(\mathrm{H}_{2} \mathrm{O}\right)}\right. \\
\left.\mathrm{m}^{-2} \mathrm{~s}^{-1}\right)\end{array}$} & Controle & $4,0 \pm 1,4 \mathrm{Aa}$ & $4,5 \pm 0,8 \mathrm{Ba}$ & $1,9 \pm 0,5 \mathrm{Ab}$ \\
\hline & $\mathrm{P}$ & $5,3 \pm 0,9 \mathrm{Ba}$ & $3,4 \pm 0,7 \mathrm{Ab}$ & $3,4 \pm 0,6 \mathrm{Bb}$ \\
\hline & $\mathrm{P}+\mathrm{Cal}$ & $4,4 \pm 1,1 \mathrm{ABa}$ & $7,1 \pm 0,9 \mathrm{Cb}$ & $4,9 \pm 1,3 \mathrm{Ca}$ \\
\hline & $\mathrm{P}+\mathrm{Cal}+\mathrm{G}$ & $6,6 \pm 1,6 \mathrm{Ca}$ & $4,7 \pm 0,5 \mathrm{Bb}$ & $3,3 \pm 0,3 \mathrm{BC}$ \\
\hline
\end{tabular}

0 s valores são médias \pm desvio padrão $(n=3)$. Médias seguidas da mesma letra maiúscula para os tratamentos (coluna), e da mesma letra minúscula para espécies (linhas), respectivamente, não diferem pelo teste de Tukey a $5 \%(n=3) . P=$ tratamento com aplicação de fósforo; $\mathrm{P}+\mathrm{Cal}=$ tratamento com aplicação de fósforo e calcário; $\mathrm{P}+\mathrm{Cal}+\mathrm{G}=$ tratamento com aplicação de fósforo, calcário e gesso agrícola. 
pH é corrigido (Rosolem et al., 2003), observou-se que, tanto para as concentraçóes de $\mathrm{C}$ quanto de $\mathrm{N}$ na profundidade de 0 $15 \mathrm{~cm}$, não houve aumento importante em relação ao tratamento controle. Aparentemente, houve uma pequena descida dos íons de nitrato para as camadas subsuperficiais do solo, como observado nas profundidades de $15-30$ e $30-45 \mathrm{~cm}$. Outra provável explicação pode estar ligada ao forte efeito da imobilização ou perda por volatilização pelo fogo desse elemento em solos degradados por pastagens na Amazônia (Luizão et al., 1999; Davidson et al., 2004). Os teores de fósforo encontrados na camada superficial dos tratamentos que sofreram calagem foram maiores pelo fato da correção da acidez do solo contribuir para aumentar a disponibilidade do fósforo no solo e, também, aumentar a eficiência dos fertilizantes fosfatados aplicados (Lopes et al., 2002). A aplicação de fontes de cálcio não alterou significativamente o teor de $\mathrm{K}$ na camada superficial, apesar de aumentar o teor de $\mathrm{Ca}$, confirmando observaçōes de experimento anterior com solos de pastagens (Wadt \& Wadt, 1999). A maior concentração de $\mathrm{K}$ observada nas camadas subsuperficiais do tratamento $\mathrm{P}+\mathrm{Cal}+\mathrm{G}(15-30$ e $30-45 \mathrm{~cm})$, pode estar relacionada à lixiviação desse elemento da camada superficial para as camadas subjacentes, favorecidas pela aplicação de gesso. As maiores concentrações de Ca e $\mathrm{Mg}$ observadas nas profundidades de 0 15 e $15-30 \mathrm{~cm}$, de modo geral, podem ter sido proporcionadas pela aplicação de calcário. Por sua vez, os maiores valores de $\mathrm{Ca}$ observados na profundidade de $30-45 \mathrm{~cm}$ do tratamento $\mathrm{P}+\mathrm{Cal}+\mathrm{G}$ podem refletir a descida de Ca por efeito da aplicação de gesso. Porém, observou-se que a presença deste nutriente não foi suficiente para elevar significativamente o $\mathrm{pH}$ nesta camada do solo. Alguns autores enfatizam o efeito da calagem na redução da concentração de micronutrientes como o Fe, Mn e Zn em solos ácidos, pois, normalmente esses elementos são considerados prejudiciais às plantas em solos com baixo $\mathrm{pH}$ (Nunes et al., 2004). Contudo, verificou-se tal efeito somente para a concentração de $\mathrm{Zn}$, na profundidade de $0-15 \mathrm{~cm}$ dos tratamentos que sofreram calagem e aplicação de fosfato. Os baixos teores de Mn observados nas camadas subsuperficiais dos tratamentos que receberam aplicação de calcário podem ser atribuídos à lixiviação de cátions para as camadas mais profundas, visto que, na camada superficial não se evidenciou tal efeito. Redução no teor de Zn do solo, após a aplicação de calcário, também foi observada por Andreotti et al. (2001) e Roque et al. (2004). Devido ao comportamento antagônico entre Zn e P, a diminuição na concentração de $\mathrm{Zn}$ pode estar relacionada à aplicação do adubo fosfatado (Biasioli, 2003; Gonçalves et al., 2005).

\section{ESTADO NUTRICIONAL DAS PLANTAS}

Os teores foliares de nutrientes das espécies estudadas revelaram que $B$. grossularioides é ma espécie muito exigente em $\mathrm{N}$, pois, apesar de não haver diferença entre os tratamentos, foi a espécie que apresentou os maiores teores de $\mathrm{N}$ entre as três espécies estudadas. Até mesmo os valores encontrados no tratamento controle para B. grossularioides foram superiores aos observados para $L$. procera e para V.japurensis nos tratamentos com aplicação de calcário e adubo fosfatado. Em estudo sobre as relaçōes das características foliares de diferentes biomas, Reich et al. (1999) também encontraram maiores teores foliares de $\mathrm{N}$ em $B$. grossularioides, comparados com os de V.japurensis. Portanto, os maiores teores foliares de $\mathrm{N}$ observados para $B$. grossularioides, podem estar associados à característica da espécie, uma vez que não foi observada diferença na concentração desse nutriente na camada superficial do solo nos diferentes tratamentos. Apesar de ter sido observado aumento nos teores de fósforo na camada
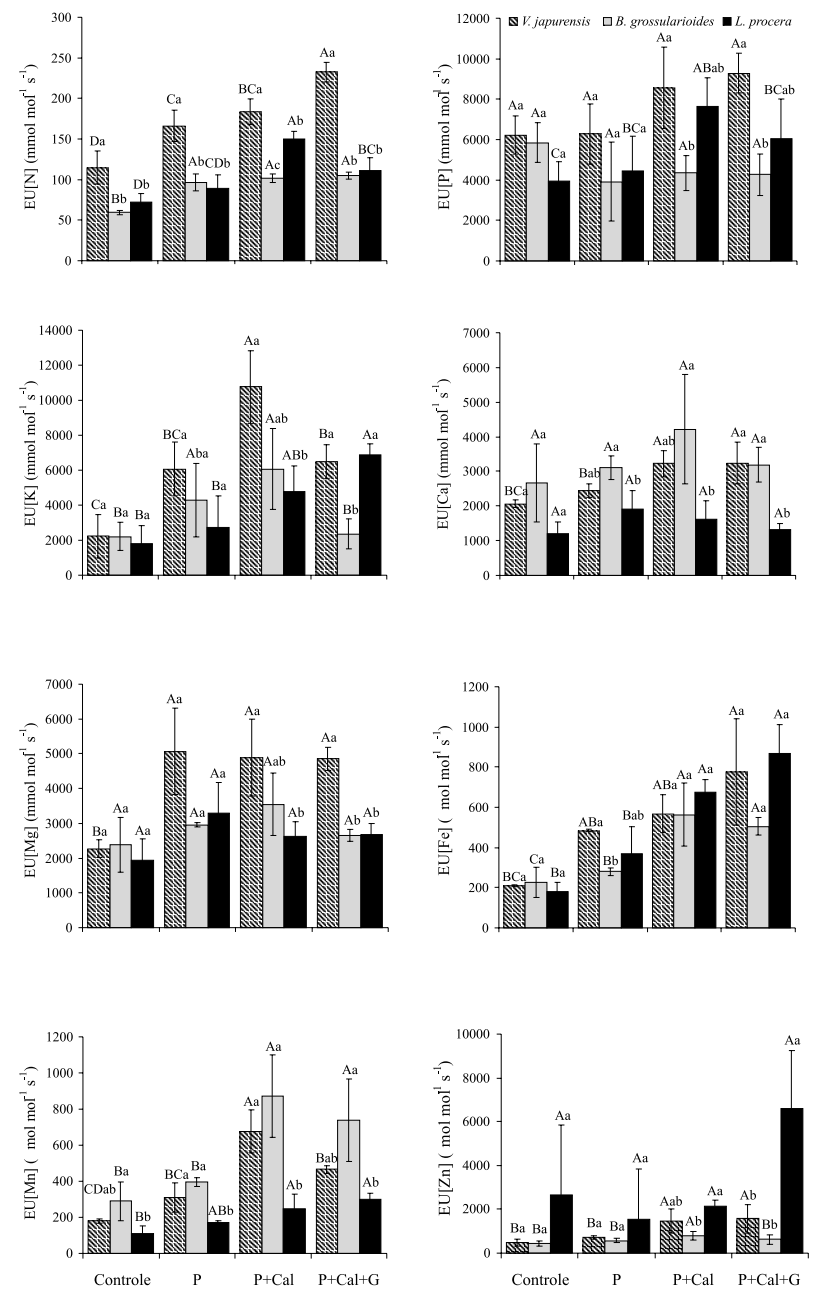

Figura 2 - Eficiência no uso dos nutrientes (EU[Nutriente]) de três espécies pioneiras, submetidas a diferentes tratamentos de correção da acidez e adubação fosfatada, numa floresta secundária, na Estação Experimental da Embrapa, na Amazônia central.

Os valores são médias $(n=3)$ e as linhas verticais indicam os desvios-padrão. Médias seguidas da mesma letra maiúscula, para os tratamentos, e da mesma letra minúscula, para espécies, não diferem pelo teste de Tukey a $5 \%$. P = tratamento com aplicação de fósforo; $\mathrm{P}+\mathrm{Cal}=$ tratamento com aplicação de fósforo e calcário; $\mathrm{P}+\mathrm{Cal}+\mathrm{G}=$ tratamento com aplicação de fósforo, calcário e gesso agrícola. 
superficial do solo dos tratamentos $\mathrm{P}$ e $\mathrm{P}+\mathrm{Cal}$ e $\mathrm{P}+\mathrm{Ca}+\mathrm{G}$, e na camada subsuperficial do tratamento $\mathrm{P}+\mathrm{Cal}+\mathrm{G}$, em função da calagem e da aplicação de adubo fosfatado, verificou-se que somente as plantas de B. grossularioides apresentaram maiores teores foliares de $\mathrm{P}$ nas plantas localizadas naqueles sítios, quando comparadas com as plantas localizadas no tratamento controle. Por serem espécies típicas de vegetação secundária e apresentarem rápido crescimento sobre áreas degradadas por pastagens, onde $o$ elemento P é sempre limitante, tanto $V$. japurensis quanto $L$. procera parecem ser pouco exigentes por esse nutriente, apesar da maior oferta proporcionada pela aplicação fosfatada. De fato, num estudo sobre a resposta da vegetaçáo secundária a diferentes tratamentos de fertilização, Gehring et al. (1999), observaram que a suspensão do nutriente $P$ não comprometeu o desempenho de V.guianensis quando comparado com o desempenho da mesma espécie no tratamento com fertilização completa $(\mathrm{N}, \mathrm{P}, \mathrm{K}, \mathrm{Ca}$, $\mathrm{Mg}, \mathrm{Fe}, \mathrm{Mn}, \mathrm{Cu}, \mathrm{Zn}, \mathrm{Mo} \mathrm{e} \mathrm{B})$. Da mesma forma, Davidson et al. (2004), em estudo sobre a limitação do crescimento da biomassa da vegetação secundária por nutrientes, não observaram aumento importante de $\mathrm{P}$ no tratamento fertilizado com fosfato em relação ao tratamento controle. O maior acúmulo de Ca observado para a espécie $L$. procera, proporcionado pela aplicação de calcário, em relação às demais espécies estudadas, pode ser considerado uma característica da espécie, uma vez que o conteúdo de Ca nas plantas é, em grande parte, controlado geneticamente e, via de regra, pouco afetado pelo suprimento de Ca no ambiente da raiz, contanto que a disponibilidade de Ca seja adequada para o crescimento normal da planta (Malavolta, 1992). Em estudo realizado por Silva et al. (1994), o efeito da calagem sobre as concentrações foliares de $\mathrm{Ca}$ e de $\mathrm{Mg}$ não acompanhou precisamente os aumentos detectados nas análises de solo. No presente estudo, os teores foliares de $\mathrm{Mg}$ foram maiores nas espécies B. grossularioides e L. procera do que em V.japurensis nos tratamentos nos tratamentos $\mathrm{P}+\mathrm{Cal}$ e $\mathrm{P}+\mathrm{Cal}+\mathrm{G}$; porém, os teores de $\mathrm{Mg}$ não diferiram entre os tratamentos estudados. Provavelmente, o crescimento dessas espécies não seja limitado pela escassez desse nutriente; tampouco, a sua presença em excesso favoreça uma maior absorção desse nutriente por plantas adaptadas a ambientes com solos tipicamente ácidos e com baixos teores desses nutrientes, conforme observado por Gehring et al. (1999), para quem somente N e P mostraram-se limitantes ao crescimento de espécies pioneiras sobre áreas degradadas na Amazônia. Outros autores também relacionam a correção da acidez do solo à redução da concentração dos micronutrientes foliares, uma vez que a absorção desses elementos pelas plantas decresce com a elevação do $\mathrm{pH}$, em decorrência da formação de óxidos e hidróxidos de baixa solubilidade (Oliveira Jr. et al., 2000; Roque et al., 2004). No entanto, para o teor foliar de Mn, não foi observado tal comportamento nas espécies estudadas. Em parte, isto pode ser explicado porque não houve uma redução na concentração desse elemento na camada superficial do solo dos tratamentos com calagem, o que favoreceu a maior absorção por essas espécies. Além disso, $L$. procera parece ser muito exigente em Mn, visto que, em todos os tratamentos estudados, apresentou maiores teores foliares. As espécies estudadas mostraram preferência pela absorçãoo de $\mathrm{Ca}^{2+}$ ao invés $\mathrm{de}^{2} \mathrm{~F}^{2+}$ com destaque para $L$. procera, que exibiu elevados teores de Ca nos tratamentos $\mathrm{P}+\mathrm{Cal}$ e $\mathrm{P}+\mathrm{Cal}+\mathrm{G}$. A redução na concentração de $\mathrm{Zn}$ na camada superficial dos tratamentos com calagem refletiu-se em menores teores foliares para $V$. japurensis e $L$. procera, nos mesmos tratamentos, o que evidencia a necessidade desse elemento nestas duas espécies, o que não foi observado para B. grossularioides, pelo menos no período de realização do experimento (oito meses). Roque et al. (2004) estudando o comportamento da seringueira, observaram decréscimo nos teores foliares de $\mathrm{Zn}$ quatorze meses após a aplicação de corretivos da acidez do solo, fato que mostra que o tempo de reação do calcário aplicado pode ter significativa importância na concentração foliar desses nutrientes.

\section{TROCAS GASOSAS}

Os resultados revelaram que a melhoria da condição edáfica proporcionada pelos tratamentos, foi importante para o aumento das taxas fotossintéticas das espécies crescendo nos tratamentos $\mathrm{P}+\mathrm{Cal}$ e $\mathrm{P}+\mathrm{Cal}+\mathrm{G}$. Via de regra, as taxas de fotossíntese líquida $(A)$ são altamente relacionadas com a taxa da condutância $(g)$, uma vez que, para absorver $\mathrm{CO}_{2}$ do meio externo, as plantas perdem água e, para diminuir estas perdas, restringem a entrada de $\mathrm{CO}_{2}$ para a interior das folhas (Lawlor, 2002). Além disso, numa tentativa de diminuir a perda de água sob altas temperaturas, as plantas diminuem a abertura estomática e a Rubisco passa a atuar como oxigenase em decorrência da redução da concentração de $\mathrm{CO}_{2}$ interno, o que de fato, causa uma redução de $50 \%$ na eficiência da fixação fotossintética do carbono (Wingler $e t$ al., 2000). Desse modo, as maiores taxas de fotorrespiração $(P$, observadas para as diferentes espécies nos tratamentos com correção da acidez e aplicação de fosfato, podem significar que importante quantidade do carbono assimilado pela fotossíntese deve estar sendo perdida na fotorrespiração. Por outro lado, a participação do processo respiratório para a economia geral de carbono nas plantas tem sido associada ao componente de perda de carbono, uma vez que grande parte do carbono assimilado na fotossíntese é utilizada pela respiração e somente parte deste carbono é efetivamente utilizada na produção de biomassa (Lambers et al., 1998). Portanto, quando se comparam as taxas de $A$ e de respiração $\left(R_{d}\right)$ para as espécies V.japurensis e L. procera, nos tratamentos controle e $\mathrm{P}+\mathrm{Cal}$, verifica-se maiores taxas de $R_{d}$ para $L$. procera, indicando que $V$. japurensis apresentou comportamento fotossintético mais eficiente. Na comparação das duas espécies com maiores taxas de $A$ ( $B$. grosularioides, no tratamento $\mathrm{P}+\mathrm{Cal}$, e $V$. japurensis, no tratamento $\mathrm{P}+\mathrm{Cal}+\mathrm{G}$ ) observa-se maiores valores das variáveis $g_{s}, R_{d}$ e $P_{r}$ para $B$. grossularioides, apesar da diferença entre essas duas espécies para a taxa de $A$ tenha sido relativamente pequena. Assim, levando-se 
em consideração que $V$. japurensis apresentou o segundo maior valor da taxa de $A$ nos tratamentos que receberam correção da acidez e aplicação de fosfato, é coerente afirmar que essa espécie apresentou maior eficiência na assimilação de $\mathrm{CO}_{2}$, menor perda de água e, conseqüentemente, maior incorporação de biomassa, se comparada às demais espécies estudadas.

\section{EFICIÊNCIA NO USO DOS NUTRIENTES}

As três diferentes espécies pioneiras, submetidas a tratamentos de correção da acidez do solo e aplicação de adubo fosfatado, de maneira geral, apresentaram melhor desempenho referente à utilização eficiente dos nutrientes. V.japurensis mostrou-se mais eficiente na utilização da maioria dos nutrientes, sendo esta maior eficiência reflexo dos altos valores da taxa fotossintética observada para essa espécie nos tratamentos com correção da acidez do solo e aplicação de fosfato $(\mathrm{P}+\mathrm{Cal}$ e $\mathrm{P}+\mathrm{Cal}+\mathrm{G})$. Apesar de terem sido observados maiores teores foliares de $\mathrm{P}$ para $B$. grossularioides nos tratamentos com aplicação de fosfato ( $\mathrm{P}, \mathrm{P}+\mathrm{Cal}$ e $\mathrm{P}+\mathrm{Cal}+\mathrm{G}), V$. japurensis utilizou de forma mais eficiente este elemento nos tratamento $\mathrm{P}+\mathrm{Cal}$ e $\mathrm{P}+\mathrm{Cal}+\mathrm{G}$ mesmo não tendo sido detectadas diferenças nos teores foliares para essa espécie entre os diferentes tratamentos, o que evidencia, de certa forma, que o $\mathrm{P}$ não é limitante para o bom desempenho desta espécie nestas áreas degradadas. Illenseer \& Paulilo (2002), estudaram plantas jovens de Euterpe edulis e verificaram que a eficiência na utilização do fósforo foi maior com o aumento da irradiância, mas não foi influenciada pelos níveis de fósforo. $V$. japurensis também se mostrou eficiente na utilização de $\mathrm{N}$ e $\mathrm{Mg}$ nos tratamentos $\mathrm{P}$, $\mathrm{P}+\mathrm{Cal}$ e $\mathrm{P}+\mathrm{Cal}+\mathrm{G}$, quando comparada com $B$. grossularioides e $L$. procera, mesmo apresentando os menores teores foliares destes nutrientes nos mesmos tratamentos. O maior acúmulo foliar de Ca e Mg pela espécie L. procera não se refletiu em maior eficiência no uso desses nutrientes, provavelmente, devido aos menores valores da taxa fotossintética observadas para essa espécie, nos tratamentos com correção da acidez e aplicação de fosfato, indicando menor demanda por esses cátions, quando comparada com as demais espécies. Apesar dos baixos valores de $\mathrm{Zn}$ verificados para L. procera nos tratamentos $\mathrm{P}+\mathrm{Cal}$ e $\mathrm{P}+\mathrm{Cal}+\mathrm{G}$, constatou-se maior eficiência na utilização deste elemento por esta espécie, quando comparada com $V$. japurensis e $B$. grossularioides. Estes resultados sugerem que as diferentes espécies que compóem o quadro sucessional sobre áreas degradadas apresentam estratégias diferenciadas quanto ao acúmulo e utilização dos nutrientes, e que nem sempre o maior acúmulo de determinado nutriente significa uma maior eficiência no seu uso pelas espécies crescendo em ambientes degradados por pastagens na Amazônia.

\section{CONCLUSÕES}

A aplicação de corretivos da acidez do solo e de adubo fosfatado favoreceu os maiores acúmulos de nutrientes foliares pelas três espécies estudadas com reflexos diretos sobre as trocas gasosas.

A melhoria do ambiente edáfico contribuiu para uma melhor performance fotossintética das espécies com destaque para $V$. japurensis e B. grossularioides que aumentaram significativamente.

$V$.japurensis, apesar de ter apresentado menores teores de nutrientes foliares comparada com $B$. grossularioides, foi a espécie que utilizou de forma mais eficiente, uma vez que apresentou elevados valores das trocas gasosas.

\section{AGRADECIMENTOS}

Os autores agradecem ao Conselho Nacional de Desenvolvimento Científico e Tecnológico - CNPq; à Fundação de Amparo a Pesquisa do Estado do Amazonas - FAPEAM; ao Experimento de Grande Escala da Biosfera-Atmosfera na Amazônia - LBA; à Embrapa Amazônia Ocidental (CPAA); e a toda a equipe do Laboratório de Fisiologia e Bioquímica Vegetal do INPA/LFBV.

\section{BIBLIOGRAFIA CITADA}

Albuquerque, J.A.; Bayer, C.; Ernani, P.R.; Mafra, A.L.; Fontana, E.C. 2003. Aplicação de calcário e fósforo e estabilidade da estrutura de um solo ácido. R. Bras. Ci. Solo, 27: 799-806.

Andreotti, M.; Souza, E.C.A.; Cruscio, C.A.C. 2001. Componentes morfológicos e produção de matéria seca de milho em função da aplicação de calcário e zinco. Scientia Agricola, 58(2): 321-327.

Biasioli, G.M. 2003. Influência do pHe da força iônica na liberação de fósforo de compostos do tipo $\mathrm{Fe}-K-\mathrm{P}$ p presentes em superfosfatos. Master's Thesis, Escola Superior de Agricultura Luiz de Queiroz, Piracicaba, São Paulo. 76pp.

Brandão, A.S.P.; Rezende, G.C.; Marques, R.W.C. 2005. Crescimento agrícola no período 1999-2004, explosão da área plantada com soja $e$ meio ambiente no Brasil. Instituto de Pesquisa Econômica Aplicada - IPEA, Rio de Janeiro. 21pp.

Davidson, E.A.; Carvalho, C.J.R.; Vieira, I.C.G.; Figueiredo, R.O.; Moutinho, P.; Ishida, F.Y.; Santos, M.T.P.; Guerrero, J.B.; Kalif, K.; Saba, R.T. 2004. Nitrogen and phosphorus limitation of biomass growth in a tropical secondary forest. Ecological Applications, 14: 150-163.

Dias Filho, M.B. 1998. Pastagens cultivadas na Amazônia oriental brasileira: processos e causas de degradação e estratégias de recuperação. In: Dias, L.E.; Mello, J.W.V. (Eds). Recuperação de áreas degradadas. Viçosa: DSO-UFV/SOBRADE. p. 135-147.

Drechsel, P.; Zech, W. 1993. Mineral nutrition of tropical trees. In: Pancel, L. (Ed.) Tropical Forestry Handbook. Vol. 1. SpringerVerlag, Berlin. p. 515-567.

EMBRAPA. 1999. Manual de análises químicas de solos, plantas e fertilizantes. Brasília: Embrapa. Solos/Embrapa Informática Agropecuária/Embrapa Comunicação para Transferência de Tecnologia. 370pp. 
Feldpausch, T.R.; Riha, S.J.; Fernandes, E.C.M.; Wandelli, E.V. 2005. Development of forest structure and leaf area in secondary forests regenerating on abandoned pastures in central Amazonia. Earth Interactions, 9: 1-21.

Feldpausch, T.R.; Rondon, M.; Fernandes, E.C.M.; Riha, S.J.; Wandelli, E.V. 2004. Carbon and nutrient accumulation in secondary forests regenerating on pastures in central Amazonia. Ecological Applications, 14(4): S164-S176.

Gehring, C.; Denich, M.; Kanashiro, M.; Vlek, P.L.G. 1999. Response of secondary vegetation in Eastern Amazonia to relaxed nutrient availability constraints. Biogeochemistry, 45: 223-241.

Gonçalves, J.F.C.; Vieira, G.; Marenco, R.A.; Ferraz, J.B.S.; Santos Júnior, U.M.; Barros, F.C. 2005. Nutritional status and specific leaf area of mahogany and tonka bean under light environments. Acta Amazonica, 35(1): 23-27.

Grant, C.A.; Flaten, D.N.; Tomasiewicz, D.J.; Sheppard, S. C. 2001. A importância do fósforo no desenvolvimento inicial da planta. Potafos - Associação Brasileira para Pesquisa da Potassa e do Fosfato. Informaçôes Agronômicas, 95: 1-5.

Illenseer, R.; Paulilo, M.T.S. 2002. Crescimento e eficiência na utilização de nutrientes em plantas jovens de Euterpe edulis Mart. sob dois níveis de irradiância, nitrogênio e fósforo. Acta Bot. Bras., 16(4): 385-394.

Köppen, W. 1948. Climatologia; versão para o espanhol de Pedro R. Hendrichs Pérez. México, Fundo de Cultura Econômica. 466pp.

Lambers, H.; Chapin III, F.S.; Pons, T.L. 1998. Plant Physiological Ecology, Springer-Verlag, New York. 540pp.

Lawlor, D.W. 2002. Limitation to photosynthesis in water-stressed leaves: stomata vs. metabolism and the role of ATP. Annals Botany, 89: 871-885.

Lopes, C.F.; Tamanini, C.R.; Monte Serrat, B.; Lima, M.R. 2002. Acidez do solo e calagem. Curitiba: Universidade Federal do Paraná, Projeto de Extensão Universitária Solo Planta. 4pp.

Lovejoy, T.E.; Bierregaard, R.O. 1990. Central Amazonian forest fragments and the minimum critical size of ecosystems project. In: A.H. Gentry, (Ed). Four neotropical rainforests. Yale University Press, New Haven. p. 60-71.

Luizão, R.C.C.; Costa, E.S.; Luizão, F.J. 1999. Mudanças na biomassa microbiana e nas transformações de nitrogênio do solo em uma sequiência de idades de pastagens após derrubada e queima da floresta na Amazônia central. Acta Amazonica, 29(1): 43-56.

Malavolta, E. 1992. Fertilizantes, corretivos e produtividade: mitos e fatos. In: Reunião Brasileira de Fertilidade do Solo e Nutrição de Plantas, 20, Piracicaba, Anais. Campinas: Fundação Cargill. p. 89-153.

Marques, E.S.; Faquin, V.; Guimarães. P.T.G. 1999. Teores foliares de nutrientes no cafeeiro (Coffea arabica L.) em resposta a calcário e gesso. Ciênc. e Agrotec., 23: 140-151.
Monaco, L.M.; Mesquita, R.C.G.; Williamson, G.B. 2003. Banco de sementes de uma floresta secundária amazônica dominada por Vismia. Acta Amazonica, 33(1): 41-52.

Nunes, F.N.; Novais, R.F.; Silva, I.R.; Gebrim, F.O.; José, J.F.B. 2004. Fluxo difusivo de ferro em solos sob influência de doses de fósforo e de níveis de acidez e umidade. R. Bras. Ci. Solo, 28: 423-429.

Oliveira Jr., J.A.; Malavolta, E.; Cabral, C.P. 2000. Efeitos do manganês sobre a soja cultivada em solo de cerrado do triângulo mineiro. Pesq. Agropec. Bras., 35(8): 1629-1636.

Parrotta, J.A. 1992. The role of plantation forest in rehabilitating degraded ecosystems. Agric. Ecosys. Environ., 41: 115-133.

Puerta, R. 2002. Regeneração arbórea em pastagens abandonadas na região de Manaus em função da distância da floresta contínua. Scientia Forestalis, 62: 32-39.

Reich, P.B.; Ellsworth, D.S.; Walters, M.B.; Vose, J.M.; Gresham, C.; Volin, J.C.; Bowman, W.D. 1999. Generality of leaf trait relationships: a test across six biomes Ecology, 80(6): 19551969.

Roque, C.G.; Prado, R.M.; Natale, W.; Beutler, A.N.; Centurion, J.F. 2004. Estado nutricional e produtividade da seringueira em solo com calcário aplicado superficialmente. Pesq. Agropec. Bras., 39(5): 485-490.

Rosolem, C.A.; Foloni, J.S.S.; Oliveira, R.H. 2003. Dinâmica do nitrogênio no solo em razão da calagem e adubação nitrogenada, com palha na superfície. Pesq. Agropec. Bras., 38(2): 301-309.

Santos Jr., U.M. 2003. Ecofisiologia de espécies arbóreas plantadas sobre áreas degradadas por atividade petrolifera na Amazônia Central. Master's Thesis, Instituto Nacional de Pesquisas da Amazônia / Fundação Universidade do Amazonas, Manaus, Amazonas. $134 \mathrm{pp}$.

Sharkey, T.D. 1988. Estimating the rate of photorespiration in leaves. Physiol. Plant., 73: 666-680.

Silva, C.A.; Vale, F.R.; Guilherme, L.R.G. 1994. Efeito da calagem na mineralização do nitrogênio em solos de Minas Gerais. $R$. Bras. Ci. Solo, 18(3): 471-476.

van Raij, B.; Andrade, J.C.; Cantarella, H.; Quaggio, J.A. 2001. Análise química para avaliação da fertilidade de solos tropicais. Instituto Agronômico. Campinas. 285 pp.

Wadt, P.G.S.; Wadt, L.H.O. 1999. Movimentação de cátions em amostras de um Latossolo Vermelho-Amarelo incubadas com duas fontes de cálcio. Scientia Agricola, 56(4): 1157-1164.

Wingler, A.; Lea, P.J.; Quick, W.P.; Leegood, R.C. 2000. Photorespiration: metabolic pathways and their role in stress protection. Phil. Trans. R. Soc. Lond., 355: 1517-1529.

Recebido em 02/08/2005

Aceito em 31/08/2006 
